\title{
Modification of the Tumor-Node-Metastasis Staging System for Differentiated Thyroid Carcinoma by Considering Extra-Thyroidal Extension and Lateral Cervical Lymph Node Metastasis
}

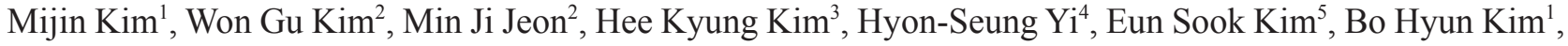
Won Bae Kim², Young Kee Shong ${ }^{2}$, Ho-Cheol Kang ${ }^{3}$, Tae Yong Kim ${ }^{2}$

${ }^{1}$ Division of Endocrinology and Metabolism, Department of Internal Medicine, Biomedical Research Institute, Pusan National University Hospital, Busan; ${ }^{2}$ Division of Endocrinology and Metabolism, Department of Internal Medicine, Asan Medical Center, University of Ulsan College of Medicine, Seoul; ${ }^{3}$ Department of Internal Medicine, Chonnam National University Medical School, Gwangju; ${ }^{4}$ Department of Internal Medicine, Chungnam National University Hospital, Chungnam National University College of Medicine, Daejeon; ${ }^{5}$ Department of Internal Medicine, Ulsan University Hospital, University of Ulsan College of Medicine, Ulsan, Korea
\end{abstract}

Background: Concerns have arisen about the classification of extra-thyroidal extension (ETE) and lateral cervical lymph node metastasis (N1b) in the 8th edition of the tumor-node-metastasis staging system (TNM-8). This study evaluated the prognostic validity of a modified-TNM staging system, focusing on ETE and N1b, in differentiated thyroid carcinoma (DTC) patients.

Methods: This multicenter retrospective cohort study included 4,878 DTC patients from five tertiary hospitals. In the modifiedTNM, T3b in TNM-8 was down-staged to T2, and stage II was subdivided into stages IIA and IIB. Older patients with N1b were reclassified as stage IIB.

Results: The modified-TNM resulted in staging migration in 540 patients (11\%) classified as stage II according to the TNM-8, with 75 (14\%), 381 (71\%), and 84 patients (16\%) classified as stages I, IIA, and IIB, respectively. The 10-year disease-specific survival (DSS) rates in patients classified as stages I, II, III, and IV by TNM- 8 were $99.8 \%, 95.9 \%, 81.0 \%$, and $41.6 \%$, respectively. The DSS rates of patients classified as stages I, IIA, IIB, III, and IV according to the modified-TNM were $99.8 \%, 96.4 \%, 93.3 \%, 81.0 \%$, and $41.6 \%$, respectively. DSS curves between stages on TNM-8 $(P<0.001)$ and modified-TNM $(P<0.001)$ differed significantly, but the modified-TNM discriminated better than TNM- 8 . The proportions of variation explained values of TNM- 8 and modified-TNM were $6.3 \%$ and $6.5 \%$, respectively.

Conclusion: Modification of the TNM staging system focusing on ETE and N1b could improve the prediction of DSS in patients with DTC. Further researches are needed to validate the prognostic accuracy of this modified-TNM staging system.

Keywords: Lymph nodes; Mortality; Neoplasm staging; Thyroid neoplasms

Received: 15 October 2019, Revised: 22 December 2019,

Accepted: 16 January 2020

Corresponding authors: Tae Yong Kim

Division of Endocrinology and Metabolism, Department of Internal Medicine,

Asan Medical Center, University of Ulsan College of Medicine, 88 Olympic-ro

43-gil, Songpa-gu, Seoul 05505, Korea

Tel: +82-2-3010-3249, Fax: +82-2-3010-6962, E-mail: tykim@amc.seoul.kr

Ho-Cheol Kang

Department of Internal Medicine, Chonnam National University Medical

School, 160 Baekseo-ro, Dong-gu, Gwangju 61469, Korea

Tel: +82-61-379-7628, Fax: +82-61-379-7620, E-mail: drkang@chonnam.ac.kr

\section{Copyright $\odot 2020$ Korean Endocrine Society}

This is an Open Access article distributed under the terms of the Creative Commons Attribution Non-Commercial License (https://creativecommons.org/ licenses/by-nc/4.0/) which permits unrestricted non-commercial use, distribution, and reproduction in any medium, provided the original work is properly cited. 


\section{INTRODUCTION}

Cancer staging plays an important role in patient management and in communications about the prognosis of disease among clinicians and between physicians and their patients [1]. Therefore, accurate tumor staging is critical for treatment decisions and ultimately for patient outcomes [1,2]. The American Joint Committee on Cancer (AJCC) tumor-node-metastasis (TNM) staging system has been widely used to predict disease mortality in patients with differentiated thyroid carcinoma (DTC) [1-3]. The revised 8th edition of the AJCC TNM staging system (TNM-8) for DTC includes important changes from the 7th edition (TNM-7) $[2,3]$ to minimize over-staging of older patients with low-risk tumors and to provide a more realistic prognosis for patients in high-risk groups [4]. Comparative studies of patients staged by the TNM- 7 and TNM- 8 showed that the changes incorporated into the TNM- 8 could lead to down-staging of approximately $40 \%$ of patients with DTC and could better predict patient mortality $[5,6]$. However, survival discrimination powers were still low in both studies and some aspects of the TNM-8 require improvement $[5,6]$.

Survival outcomes in DTC patients with gross extra-thyroidal extension (ETE) were found to differ according to the direction and extent of ETE $[7,8]$. Recently, a single center study showed that disease-specific survival (DSS) was better in patients with stage T3b DTC (gross ETE invading only the strap muscles with tumor size $\leq 4 \mathrm{~cm}$ ) than for patients with T3a DTC (tumor size $>4 \mathrm{~cm}$ and limited to the thyroid), with the former having DSS similar to that of patients with T2 DTC [7]. These results suggested the importance of downgrading stage T3b to stage $\mathrm{T} 2$ [7]. In addition, modifying TNM-8 by employing N1b classification may better predict survival outcomes $[9,10]$. The present study investigated whether the TNM-8 classification could be improved by including ETE and N1b in the evaluation of patients with DTC. Specifically, this study compared the prognostic ability of the TNM-8 staging system and a modification of this staging system that included gross ETE and lateral cervical lymph node (LN) metastasis (N1b) to predict DSS in a large, multicenter cohort of patients with DTC.

\section{METHODS}

\section{Patients}

In this retrospective, multicenter cohort study, 4,878 patients with DTC who underwent thyroid surgery between 1996 and 2005 at five tertiary hospitals in Korea were included: Asan
Medical Center $(n=3,176)$, Chonnam National University Hwasun Hospital $(n=691)$, Pusan National University Hospital $(n=552)$, Ulsan University Hospital $(n=345)$, and Chungnam National University Hospital $(n=114)$. All the included patients were pathologically proven to have papillary thyroid carcinoma (PTC) or follicular thyroid carcinoma (FTC). The research protocol was approved by the Institutional Review Board of each institution: Asan Medical Center, 2016-1301; Chonnam National University Hwasun Hospital, CNUHH-2017-053; Pusan National University Hospital, 1701-014-051; Ulsan University Hospital, 2016-12-031; and Chungnam National University Hospital, CNUH 2017-01-018. Informed consent was waived due to the retrospective design of this study [10].

The clinical and pathological data obtained from electronic medical records included age at the time of diagnosis, sex, primary tumor size, pathologic subtype, extent of ETE, presence and location of cervical LN metastases, presence of distant metastases, extent of surgery, and use of radioactive iodine ablation. The survival status, last follow-up time, and causes of death were also collected.

\section{Definition of modified-TNM}

Patients categorized according to the TNM-8 [3] were subsequently reclassified using a modified-TNM staging system (Table 1). Patients were reclassified into different $T$ and TNM stages by the modified-TNM staging system. Patients classified as T3b (gross ETE invading only strap muscles with tumor size $\leq$ $4 \mathrm{~cm}$ ) according to the TNM-8 were reclassified as T2 according to the modified-TNM, and stage II on the TNM- 8 was subdivided into stages IIA and IIB on the modified-TNM. Older patients ( $\geq 55$ years) with $\mathrm{N} 1 \mathrm{~b}$ disease were reclassified as stage IIB on the modified-TNM, whereas the remaining patients were classified into stage IIA.

\section{Primary outcome}

The TNM staging system is useful for predicting disease mortality and therefore recommended for staging of patients with DTC [3]. Therefore, the primary outcome of this study was DSS, defined as the time interval from the date of the initial thyroidectomy to the date of last censor or death due to DTC.

\section{Statistics}

Data were analyzed using R version 3.5.1 software and the R libraries (R Foundation for Statistical Computing, Vienna, Austria; http://www.R-project.org). Categorical variables were presented as numbers with percentages and continuous variables 
Table 1. Prognostic Stages and Stage Groups According to the TNM-8 and the Modified-TNM TNM-8 Modified-TNM

\section{T category}

$\begin{array}{ll}\text { TX } & \text { Primary tumor cannot be assessed } \\ \text { T0 } & \text { No evidence of primary tumor } \\ \text { T1 } & \text { Tumor size } \leq 2 \mathrm{~cm}, \text { limited to the thyroid } \\ \text { T2 } & \text { Tumor size }>2 \mathrm{~cm} \text { and } \leq 4 \mathrm{~cm}, \text { limited to the thyroid }\end{array}$

T3a

$\mathrm{T} 3 \mathrm{~b}$

$\mathrm{T} 4 \mathrm{a}$

$\mathrm{T} 4 \mathrm{~b}$
Tumor size $>4 \mathrm{~cm}$, limited to the thyroid

Gross extrathyroidal extension invading only strap muscles from a tumor of any size

Gross extrathyroidal extension invading subcutaneous soft tissues, larynx, trachea, esophagus, or recurrent laryngeal nerve
Gross extrathyroidal extension invading preverte
casing the carotid artery, or mediastinal vessels

\author{
Same as 8 th edition \\ Same as 8 th edition \\ Same as 8 th edition \\ Tumor size $>2 \mathrm{~cm}$ and $\leq 4 \mathrm{~cm}$, limited to the thyroid, or gross \\ extrathyroidal extension invading only strap muscles with \\ tumor size $\leq 4 \mathrm{~cm}$ \\ Tumor size $>4 \mathrm{~cm}$, limited to the thyroid \\ with tumor size $>4 \mathrm{~cm}$ \\ Same as 8 th edition \\ - Same as 8 th edition
}

The definitions of $\mathrm{N}$ and $\mathrm{M}$ categories are identical

Stage group

$$
\begin{aligned}
& \text { I: Tx Nx M0 }<55 \text { yr } \\
& \text { T1-2 N0/NX M0 } \geq 55 \text { yr } \\
& \text { II: Tx Nx M1 }<55 \text { yr } \\
& \text { T1-2 N1 M0 } \geq 55 \text { yr } \\
& \text { T3a/T3b Nx M0 } \geq 55 \text { yr }
\end{aligned}
$$

III: T4a Nx M0 $\geq 55$ yr

IVA: T4b Nx M0 $\geq 55$ yr

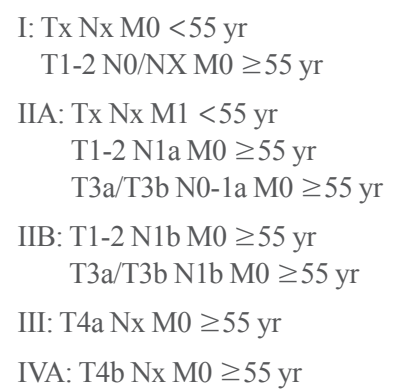

TNM-8, 8th edition of the AJCC TNM staging system; TNM, tumor-node-metastasis.

were as means with standard deviations. We plotted DSS curves using the Kaplan-Meier method and compared them using logrank test. We evaluate the impact of TNM stage groupings on DSS based on the Cox-proportional hazard model. Relative risks of survival are reported as hazard ratios (HRs), 95\% confidence interval (CI), and $P$ value.

The proportion of variation explained (PVE) in the Cox-proportional hazard model was calculated to estimate how well each of the staging systems predicted the outcome of DSS. PVE, which ranges from 0 to 100 , was calculated using the formula: $\mathrm{PVE}=1-\exp \left(-\mathrm{G}^{2} / n\right)$, in which $\mathrm{G}^{2}$ is the maximum likelihood ratio determined by analysis using the chi-square test associated with the null hypothesis, and $n$ is the total number of valid patients in the study [11]. All $P$ values were two-sided, and $P$ values less than 0.05 were considered statistically significant.

\section{RESULTS}

\section{Baseline characteristics}

The baseline clinical and pathological characteristics of the 4,878 patients with DTC are illustrated in Table 2. Mean patient age was $45.9 \pm 12.2$ years, and $87 \%$ were women. Most patients $(n=4,704,96 \%)$ were diagnosed with PTC. The mean primary tumor size was $1.7 \pm 1.3 \mathrm{~cm}$, and 3,534 (72\%), 1,116 (23\%), and 228 patients (5\%) had primary tumor sizes $\leq 2,2$ to 4 , and $>4$ $\mathrm{cm}$, respectively. Of the 4,878 patients, 1,407 (29\%), 577 (12\%), and $267(5 \%)$ showed microscopic ETE, gross ETE invading the strap muscles alone, and gross ETE invading areas other than the strap muscles, respectively. Of the 577 patients with gross ETE invading only the strap muscles, $530(92 \%)$ had primary tumors $\leq 4 \mathrm{~cm}$ in size and $47(8 \%)$ had tumors $>4 \mathrm{~cm}$. Cervical LN metastases were identified in 2,116 patients (43\%), including 1,624 (33\%) with N1a and $492(10 \%)$ with N1b dis- 
Table 2. Baseline Clinical and Pathological Characteristics of Patients with Differentiated Thyroid Carcinoma

\begin{tabular}{lc}
\hline Characteristic & Value \\
\hline Number & 4,878 \\
Age, yr & $45.9 \pm 12.2$ \\
Female sex & $4,226(87)$ \\
Pathology & \\
PTC & $4,704(96)$ \\
FTC & $174(4)$ \\
Primary tumor size, cm & $1.7 \pm 1.3$ \\
$\leq 2$ & $3,534(72)$ \\
$2-4$ & $1,116(23)$ \\
$>4$ & $228(5)$ \\
Extra-thyroidal extension & \\
Microscopic & $1,407(29)$ \\
Gross, invading only strap muscle & $577(12)$ \\
$\quad$ With primary tumor size $\leq 4$ cm & 530 \\
$\quad$ With primary tumor size $>4$ cm & 47 \\
Gross, invading other than strap muscle & $267(5)$ \\
Cervical LN metastasis & \\
N1a & \\
N1b & \\
Distant metastasis & $3,624(33)$ \\
Total thyroidectomy & $3,140(64)$ \\
RAI treatment & \\
\hline Values are expressed as mean \pm standard deviation or number $(\%)$ \\
PTC, papillary thyroid carcinoma; FTC, follicular thyroid carcinoma; \\
LN, lymph node; RAI, radioactive iodine. \\
$\quad$ \\
\hline
\end{tabular}

ease. Distant metastasis at diagnosis was found in 74 patients (2\%). The median follow-up period until censoring or death was 10.2 years (interquartile range, 7.8 to 12.0 ).

\section{Distribution of patients per stage according to the TNM-8 and the modified-TNM}

Fig. 1 shows the migration of staging from the TNM- 8 to the modified-TNM. Of 709 patients classified as having T3 disease on the TNM-8, 530 (75\%) with T3b (gross ETE invading only strap muscles with tumor size $\leq 4 \mathrm{~cm}$ ) disease were down-graded to the T2 category (Fig. 1A). The TNM-8 classified 4,214 patients (86\%) as having stage I tumors, $540(11 \%)$ as having stage II, 97 (2\%) as having stage III, and $27(1 \%)$ as having stage VI (Fig. 1B). Application of the modified-TNM to the 540 patients classified as stage II according to the TNM-8 resulted in $75(14 \%)$ of these patients being down-staged to stage I, with
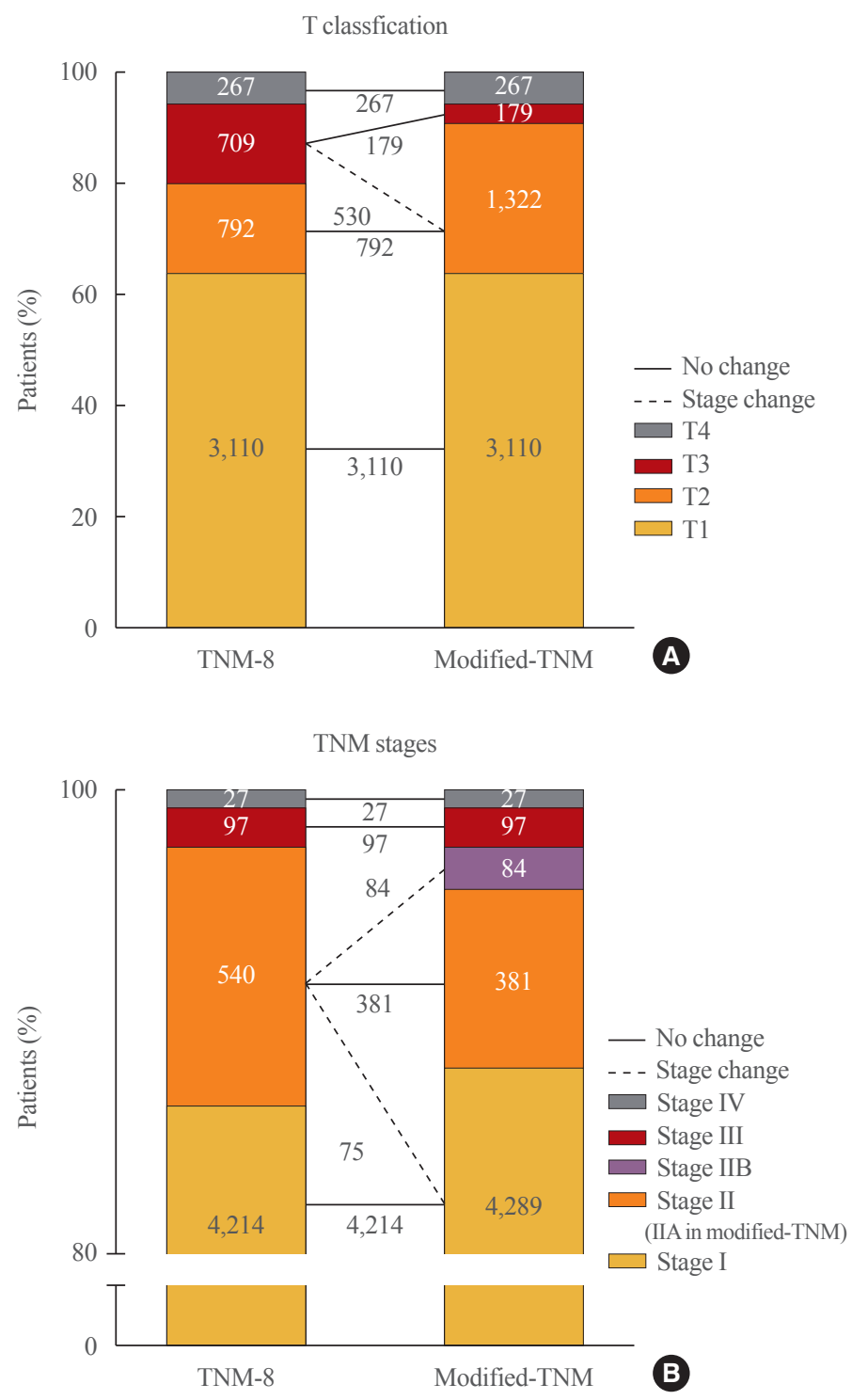

Fig. 1. Distribution of patients according to (A) T classifications and (B) tumor-node-metastasis (TNM) stages, as determined by the 8th edition of the TNM staging system (TNM-8) and the modifiedTNM staging system.

the remaining $381(71 \%)$ and 84 patients (16\%) reclassified as stages IIA and IIB, respectively. Among the 75 patients being down-staged to stage I, there were 72 classical PTC, two follicular variant PTC, and one FTC. Patients with stages III and IV tumors according to the TNM- 8 showed no changes when classified according to the modified-TNM.

\section{DSS according to the TNM-8 and modified-TNM}

DSS in patients with DTC was evaluated according to the TNM-8 and modified-TNM staging systems (Table 3, Fig. 2). 
Table 3. Relative Risk of DSS and Prognostic Validity According to the TNM-8 and the Modified-TNM

\begin{tabular}{|c|c|c|c|c|c|c|}
\hline Variable & No. $(\%)$ & 10 -yr DSS rates, $\%$ & HR & $95 \% \mathrm{CI}$ & $P$ value & PVE, \% \\
\hline TNM-8 & & & & & & 6.3 \\
\hline Stage I & $4,214(86)$ & 99.8 & 1.0 & Reference & & \\
\hline Stage II & $540(11)$ & 95.9 & 23.0 & $11.6-45.6$ & $<0.001$ & \\
\hline Stage III & $97(2)$ & 81.0 & 143.0 & $68.8-297.5$ & $<0.001$ & \\
\hline Stage IV & $27(1)$ & 41.6 & 366.7 & $173.8-744.0$ & $<0.001$ & \\
\hline Modified-TNM & & & & & & 6.5 \\
\hline Stage I & $4,289(88)$ & 99.8 & 1.0 & Reference & & \\
\hline Stage IIA & $381(8)$ & 96.4 & 16.7 & $8.1-34.0$ & $<0.001$ & \\
\hline Stage IIB & $84(2)$ & 93.3 & 38.6 & $17.4-85.4$ & $<0.001$ & \\
\hline Stage III & $97(2)$ & 81.0 & 121.1 & $60.5-242.5$ & $<0.001$ & \\
\hline Stage IV & $27(1)$ & 41.6 & 313.2 & $154.1-636.8$ & $<0.001$ & \\
\hline
\end{tabular}

DSS, disease-specific survival; TNM-8, 8th edition of the AJCC TNM staging system; TNM, tumor-node-metastasis; HR, hazard ratio; CI, confidence interval; PVE, proportion of variation explained.

TNM-8

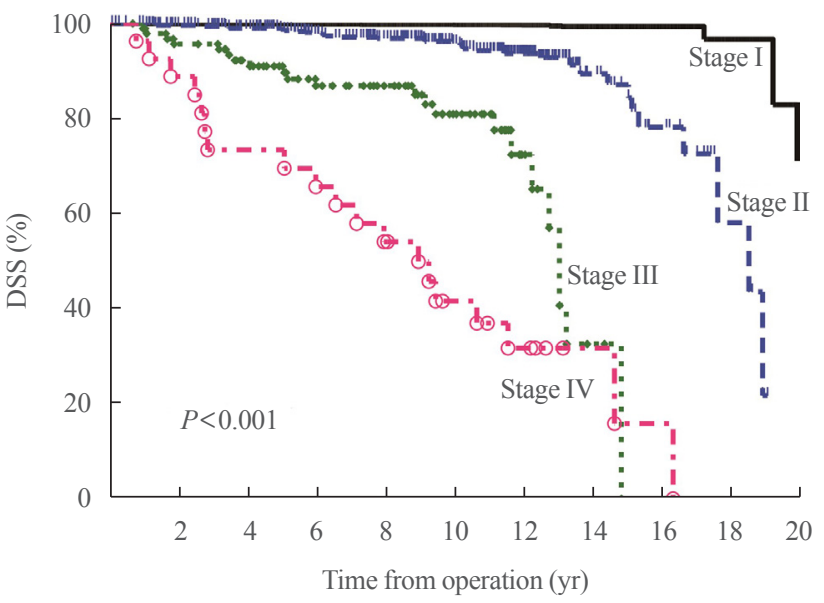

Modified-TNM

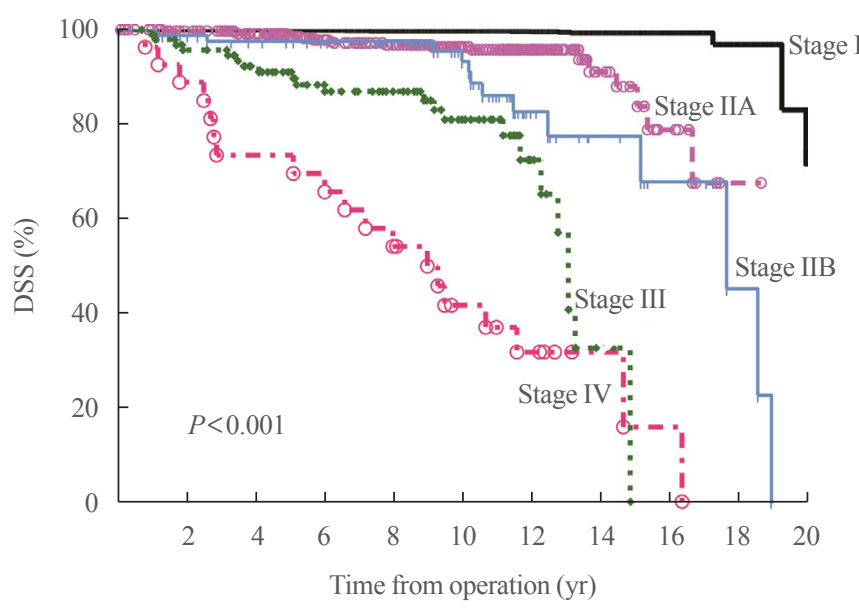

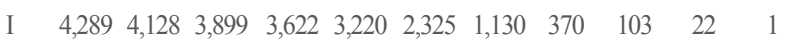

$\begin{array}{llllllllllll}\text { IIA } & 381 & 357 & 337 & 290 & 263 & 181 & 97 & 33 & 10 & 1 & 0\end{array}$

$\begin{array}{llllllllllll}\text { IIB } & 84 & 79 & 74 & 69 & 62 & 42 & 18 & 9 & 5 & 2 & 0\end{array}$

III $\quad \begin{array}{lllllllllll}97 & 88 & 76 & 63 & 53 & 33 & 12 & 2 & 0 & 0 & 0\end{array}$

$\begin{array}{llllllllllllll}\text { A } & \text { IV } & 27 & 23 & 19 & 18 & 15 & 9 & 6 & 2 & 1 & 0 & 0 & \text { B }\end{array}$

Fig. 2. Kaplan-Meier analysis of disease-specific survival (DSS) in patients with differentiated thyroid carcinoma classified according to (A) the 8 th edition of the tumor-node-metastasis (TNM) staging system (TNM-8) and (B) the modified-TNM staging system.

DSS was associated significantly with stage at diagnosis, as determined by the TNM-8 $(P<0.001)$ (Fig. 2A) and modifiedTNM $(P<0.001)$ (Fig. 2B) staging systems. However, differences in DSS between stage groups appeared to be more distinct when patients were staged by the modified-TNM than the TNM-8 (Fig. 2).

When staged according to the TNM-8, the 10-year DSS rates for patients with stages I, II, III, and IV DTCs were 99.8\%, $95.9 \%, 81.0 \%$, and $41.6 \%$, respectively (Table 3 ). When staged according to the modified-TNM, the 10-year DSS rates for patients with stages I, IIA, IIB, III, and IV DTCs were 99.8\%, $96.4 \%, 93.3 \%, 81.0 \%$, and $41.6 \%$, respectively. Using modified-TNM stage I as the reference, patients with stage IIA (HR, 16.7; $95 \%$ CI, 8.1 to $34.0 ; P<0.001)$ and stage IIB (HR, 38.6; 

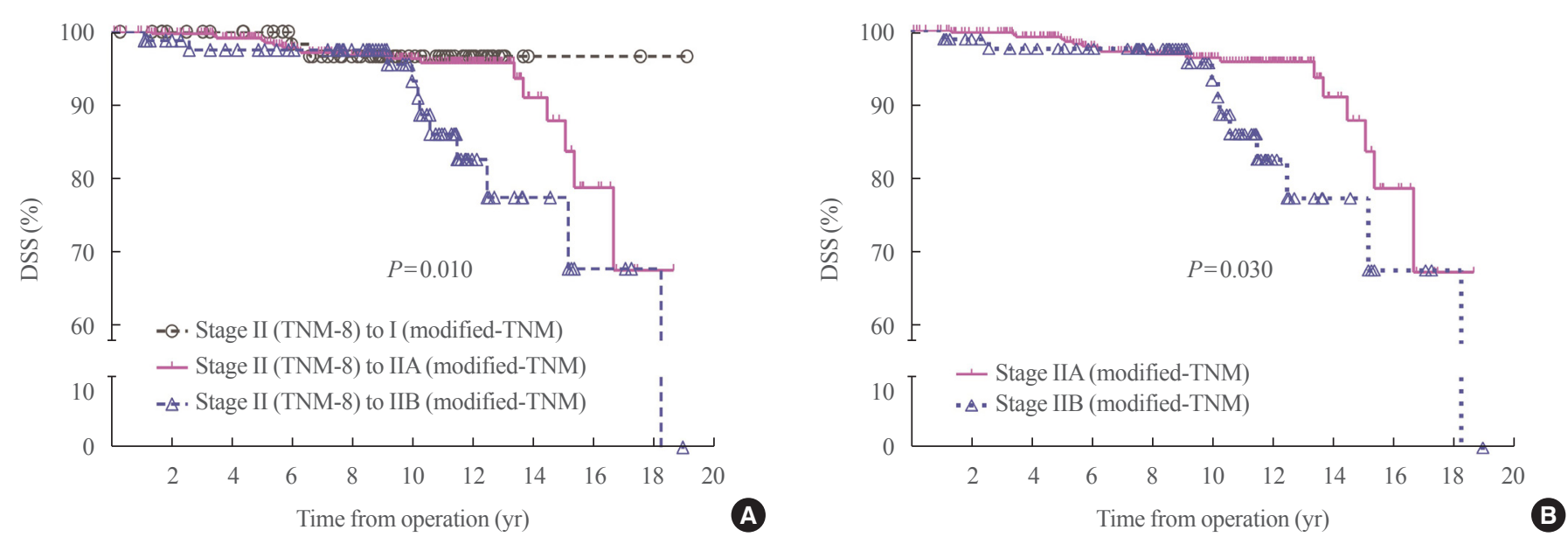

Fig. 3. Kaplan-Meier analysis of disease-specific survival (DSS) in patients who showed (A) stage migration after applying the modified-tumor-node-metastasis (TNM) staging system and (B) patients classified as stages IIA and IIB according to the modified-TNM. TNM-8, the 8th edition of the TNM staging system.

$95 \%$ CI, 17.4 to $85.4 ; P<0.001)$ had significantly poorer DSS. The value of the PVE of the modified-TNM system was $6.5 \%$ and that of the TNM- 8 was $6.3 \%$.

\section{DSS in patients with stage migration after applying the modified-TNM}

Patients classified as stage II according to the TNM-8 were reclassified as stages I, IIA, and IIB according to the modifiedTNM. Following stage migration, the DSS curves of these three groups differed significantly $(P=0.010)$ (Fig. 3A), with their 10-year DSS rates being $96.7 \%, 96.4 \%$, and $93.3 \%$, respectively. DSS rates also differed significantly between stages IIA and IIB $(P=0.030)$ (Fig. 3B), with patients classified as stage IIB having a 2.3-fold poorer DSS than those reclassified as stage IIA ( $95 \%$ CI, 1.1 to $4.8 ; P=0.035)$.

\section{DISCUSSION}

This multicenter cohort study evaluated whether and how the prognostic predictability of the TNM-8 staging system for patients with DTC could be improved by modifications focusing on both gross ETE and lateral cervical LN metastasis. Application of the modified-TNM staging system resulted in stage migration only in patients classified as stage II according to the TNM-8. About $14 \%$ of these patients were down-staged to stage I, whereas $71 \%$ and $16 \%$ were reclassified as stages IIA and IIB, respectively. The modified-TNM staging system appeared to better discriminate among stages and to better predict DSS than the TNM-8. Potential modifications, including the downgrading of patients with T3b (gross ETE invading only strap muscles with tumor size $\leq 4 \mathrm{~cm}$ ) to T2 and the upgrading of older patients with $\mathrm{N} 1 \mathrm{~b}$ disease from stage II, may better predict DSS in patients with DTC.

In the modified-TNM, tumors with gross ETE measuring $\leq 4$ $\mathrm{cm}$ are down-staged to T2 disease. This change is based on the findings of previous studies, which showed that gross ETE invading only the strap muscles did not affect survival outcome in patients with DTC, especially in patients with tumors $\leq 4 \mathrm{~cm}$ in size $[7,8,12]$. In addition, patients with gross ETE invading only the strap muscles and tumor size $\leq 4 \mathrm{~cm}$ showed no difference in DSS compared to patients with T2 disease, but rather showed significantly better DSS compared to patients with T3a disease [7]. ETE has been considered as a prognostic factor in patients with DTC and is observed in 5\% to $45 \%$ of these patients [13]. Beginning with the sixth edition of the AJCC TNM staging system, the presence of ETE has been divided into two grades, minimal and massive ETE, affecting the T category [2]. Many studies have shown that DTC patients with massive ETE have a much poorer survival outcome than those with minimal ETE $[13,14]$, and that DTC patients with minimal and no ETE have similar survival outcomes $[15,16]$. For this reason, the revised TNM-8 ascribed high importance to gross ETE but eliminated minimal ETE in T staging [3]. However, it was unclear whether patients having tumors $\leq 4 \mathrm{~cm}$ with gross ETE invading the strap muscles alone also have a poor prognosis, and whether it is appropriate for these patients to be upgraded to a higher $\mathrm{T}$ 
grade. Although $75 \%$ of patients in this study with T3 disease were down-graded to T2, the 10-year DSS rate of patients classified as stage T2 by the modified-TNM was excellent (98.9\%, data not shown).

The TNM-8 classification of patients with DTC does not consider the location of metastatic LNs in stage grouping [3]. Consequently, the TNM-8 downstages a large number of patients with $\mathrm{N} 1 \mathrm{~b}$ disease. However, there is evidence that $\mathrm{N} 1 \mathrm{~b}$ disease is a significant prognostic factor in patients with DTC, and that survival outcomes are worse for DTC patients with N1b than with N1a $[9,17,18]$. This study found that older patients with N1b disease were predicted to have a significantly lower 10year DSS rate than those with N0 or N1a disease, emphasizing the prognostic significance of N1b in older patients with DTC, and suggesting that $\mathrm{N} 1 \mathrm{~b}$ should not be treated as equivalent to N0 or N1a. Further research is needed to assess the prognostic value of N1b disease according to the modified-TNM staging system for patients with DTC.

This study had limitations because of its retrospective design. There may be variations in the intensity of treatment and the frequency of follow-up. However, our findings are based on data from a large multicenter cohort population with a relevant number of disease-specific death events during a median 10.2 years of follow-up.

In conclusion, this study provided valuable information about the suitability of the TNM-8 for staging DTC and suggested that modifications based on ETE and N1b may improve its accuracy. Differences in DSS between each pair of stages on the modified-TNM appeared greater than those between each pair of stages on the TNM-8. In addition, the modified-TNM grouping appeared to be a better predictor of DSS than the TNM- 8 . These findings indicate that the modified-TNM grouping proposed here may better predict outcomes and improve treatment selection for patients with DTC. Further researches are needed to valid the prognostic accuracy of these modifications.

\section{CONFLICTS OF INTEREST}

No potential conflict of interest relevant to this article was reported.

\section{ACKNOWLEDGMENTS}

This work was supported by the Korean Endocrine Society of EnM Research Award 2018.

\section{AUTHOR CONTRIBUTIONS}

Conception or design: M.K., W.G.K., M.J.J., H.C.K., T.Y.K. Acquisition, analysis, or interpretation of data: M.K., M,J.J., H.S.Y., E.S.K., H.C.K. Drafting the work or revising: M.K., T.Y.K. Final approval of the manuscript: M.K., W.G.K., M.J.J., H.K.K., H.S.Y., E.S.K., B.H.K., W.B.K., Y.K.S., H.C.K., T.Y.K.

\section{ORCID}

Mijin Kim https://orcid.org/0000-0002-1538-8859

Tae Yong Kim https://orcid.org/0000-0003-4982-4441

Ho-Cheol Kang https://orcid.org/0000-0002-0448-1345

\section{REFERENCES}

1. Haugen BR, Alexander EK, Bible KC, Doherty GM, Mandel SJ, Nikiforov YE, et al. 2015 American Thyroid Association Management Guidelines for adult patients with thyroid nodules and differentiated thyroid cancer: the American Thyroid Association Guidelines task force on thyroid nodules and differentiated thyroid cancer. Thyroid 2016;26:1133.

2. Edge SB, Compton CC. The American Joint Committee on Cancer: the 7th edition of the AJCC cancer staging manual and the future of TNM. Ann Surg Oncol 2010;17:1471-4.

3. Edge SB; American Joint Committee on Cancer. AJCC cancer staging manual. 8th ed. New York: Springer International Publishing; 2017.

4. Tuttle RM, Haugen B, Perrier ND. Updated American Joint Committee on cancer/tumor-node-metastasis staging system for differentiated and anaplastic thyroid cancer (Eighth edition): what changed and why? Thyroid 2017;27:751-6.

5. Kim M, Kim WG, Oh HS, Park S, Kwon H, Song DE, et al. Comparison of the seventh and eighth editions of the American Joint Committee on cancer/union for international cancer control tumor-node-metastasis staging system for differentiated thyroid cancer. Thyroid 2017;27:1149-55.

6. Kim TH, Kim YN, Kim HI, Park SY, Choe JH, Kim JH, et al. Prognostic value of the eighth edition AJCC TNM classification for differentiated thyroid carcinoma. Oral Oncol 2017;71:81-6.

7. Song E, Lee YM, Oh HS, Jeon MJ, Song DE, Kim TY, et al. A relook at the $\mathrm{T}$ stage of differentiated thyroid carcinoma with a focus on gross extrathyroidal extension. Thyroid 2019; 29:202-8. 
8. Park SY, Kim HI, Kim JH, Kim JS, Oh YL, Kim SW, et al. Prognostic significance of gross extrathyroidal extension invading only strap muscles in differentiated thyroid carcinoma. Br J Surg 2018;105:1155-62.

9. Kim M, Jeon MJ, Oh HS, Park S, Song DE, Sung TY, et al. Prognostic implication of N1b classification in the eighth edition of the tumor-node-metastasis staging system of differentiated thyroid cancer. Thyroid 2018;28:496-503.

10. Kim M, Kim HK, Kim HI, Kim EH, Jeon MJ, Yi HS, et al. Modification of the eight-edition tumor-node-metastasis staging system with N1b for papillary thyroid carcinoma: a multi-institutional cohort study. Oral Oncol 2018;86:48-52.

11. Schemper M, Stare J. Explained variation in survival analysis. Stat Med 1996;15:1999-2012.

12. Amit M, Boonsripitayanon M, Goepfert RP, Tam S, Busaidy NL, Cabanillas ME, et al. Extrathyroidal extension: does strap muscle invasion alone influence recurrence and survival in patients with differentiated thyroid cancer? Ann Surg Oncol 2018;25:3380-8.

13. Ortiz S, Rodriguez JM, Soria T, Perez-Flores D, Pinero A, Moreno J, et al. Extrathyroid spread in papillary carcinoma of the thyroid: clinicopathological and prognostic study.
Otolaryngol Head Neck Surg 2001;124:261-5.

14. Andersen PE, Kinsella J, Loree TR, Shaha AR, Shah JP. Differentiated carcinoma of the thyroid with extrathyroidal extension. Am J Surg 1995;170:467-70.

15. Ito Y, Tomoda C, Uruno T, Takamura Y, Miya A, Kobayashi $\mathrm{K}$, et al. Prognostic significance of extrathyroid extension of papillary thyroid carcinoma: massive but not minimal extension affects the relapse-free survival. World J Surg 2006;30: 780-6.

16. Woo CG, Sung CO, Choi YM, Kim WG, Kim TY, Shong YK, et al. Clinicopathological significance of minimal extrathyroid extension in solitary papillary thyroid carcinomas. Ann Surg Oncol 2015;22 Suppl 3:S728-33.

17. Nixon IJ, Wang LY, Palmer FL, Tuttle RM, Shaha AR, Shah JP, et al. The impact of nodal status on outcome in older patients with papillary thyroid cancer. Surgery 2014;156:13746.

18. Adam MA, Pura J, Goffredo P, Dinan MA, Reed SD, Scheri RP, et al. Presence and number of lymph node metastases are associated with compromised survival for patients younger than age 45 years with papillary thyroid cancer. J Clin Oncol 2015;33:2370-5. 